\title{
O experimento da borracha quântica: discutindo o quântico pelo clássico em sala de aula
}

Luciano Slovinscki ${ }^{1}$

Doutorando em Ensino de Física pelo Instituto de Física - UFRGS

Alan Alves-Brito ${ }^{1}$

Instituto de Física - UFRGS

Porto Alegre - RS

\section{Resumo}

O presente trabalho é voltado ao professor da Educação Básica, mas pode também atender aos interesses da Formação Inicial e Continuada de Professores, e propõe uma atividade que abrange duas áreas que carecem de maior atenção no Ensino de Física atual: a inserção de tópicos de Física Moderna e Contemporânea no Ensino Médio, bem como a proposição de novas atividades experimentais àquele segmento da Educação Básica. Nesse sentido, o experimento denominado "borracha quântica" aborda, qualitativamente, alguns dos princípios fundamentais da Mecânica Quântica. O experimento é composto, basicamente, de um laser e de alguns filtros polarizadores, obtidos a partir da tela de mostradores digitais usados, como calculadoras e multímetros, e permite a contextualização e a discussão dos conceitos quânticos de função de onda, estados quânticos, superposição de estados, probabilidade, determinação do estado e colapso da função de onda, segundo a interpretação da Escola de Copenhague. Os conceitos propostos, baseados nos postulados da Mecânica Quântica, passaram por um processo de transposição didática, de modo a se adaptarem ao nivel cognitivo dos estudantes do Ensino Médio. O aparato experimental é de fácil construção e utiliza materiais de baixo custo, sendo bastante viável sua exploração em sala de aula pelo professor. Os resultados obtidos experimentalmente foram considerados de boa qualidade $e$

\footnotetext{
${ }^{+}$The quantum eraser experiment: discussing quantum by classic in the classroom

* Recebido: 17 de agosto de 2020. Aceito: 27 de fevereiro de 2021.

${ }^{1}$ E-mails: 1slovinscki@gmail.com; alves.brito.a@gmail.com
} 
condizentes com o esperado, quando da aplicação do experimento a uma turma mista de estudantes do Ensino Médio.

Palavras-chave: Ensino de Física; Atividades Experimentais; Mecânica Quântica; Borracha Quântica.

\begin{abstract}
The present work is directed to the Basic Education teacher, but it can also serve the interests of Initial and Continuing Teacher Education, and proposes an activity covering two fields that require more attention in current Physics Teaching: the insertion of Modern and Contemporary Physics topics at High School, and the proposition of new experimental activities to that segment of Basic Education. The experiment called "quantum eraser" addresses, qualitatively, some of the fundamental principles of Quantum Mechanics. The experiment is composed of a laser and some polarizing filters, obtained from the screen of used digital displays, such as calculators and multimeters, and allows contextualization and discussion of the quantum concepts of wave function, quantum states, superposition of states, probability, state determination and collapse of the wave function, according to the Copenhagen School interpretation. The proposed concepts, based on the postulates of Quantum Mechanics, were subject to a didactic transposition process, to adapt to the cognitive level of high school students. The experimental apparatus is easy to build using low-cost materials, making it possible to explore it in the classroom by the teacher. The results obtained experimentally were considered of good quality and consistent with expectations, when the experiment was applied to high school students.
\end{abstract}

Keywords: Physics Teaching; Experimental Activities; Quantum Mechanics; Quantum Eraser.

\title{
I. Introdução
}

No início do século XX, os trabalhos de Max Planck (FELDENS; DIAS; SANTOS, 2010) e Albert Einstein (ARRUDA; VILLANI, 1996) marcaram a ruptura do pensamento científico tradicional que constituía a Física até então. Nascia, naquele momento, o que hoje conhecemos por Física Moderna e Contemporânea (FMC). Desde então, temos mais de um século de pesquisas nessa área de concentração e incontáveis avanços tecnológicos, fruto das 
novas teorias. Apesar disso, o ensino de Física formal contemplava, até poucos anos atrás, a quase totalidade de seus currículos apenas com assuntos de Física Clássica (FC) (OSTERMANN; MOREIRA, 2000). Verificou-se, principalmente nas últimas duas décadas, uma tentativa de mudança curricular, muito em função dos documentos que regulam a Educação no Brasil e dos editais do Programa Nacional do Livro Didático (PNLD), que recomendam fortemente a inserção de tópicos de FMC no Ensino Médio (EM). Além disso, a nova Base Nacional Comum Curricular (BNCC) reforça essa mudança nos currículos, principalmente no tocante ao EM, ao fomentar, ainda que de maneira não ideal, o ensino da FMC no EM (MOZENA; OSTERMANN, 2016).

A inserção de assuntos relacionados à FMC nos ambientes formais de ensino é algo defendido por pesquisadores da área de Ensino de Física há bastante tempo. Os trabalhos de Gil, Senent e Solbes (1988), Arons (1990), Fischler e Lichtfeldt (1992), Terrazzan (1992; 1994), Ostermann e Moreira (2000), e Greca, Moreira e Herscovitz (2001), entre outros, defendem tal ideia. Além disso, documentos como os Parâmetros Curriculares Nacionais (BRASIL, 1999) e as Orientações Educacionais Complementares aos Parâmetros Curriculares Nacionais (BRASIL, 2002), além das Orientações Curriculares para o Ensino Médio (BRASIL, 2006) e das Diretrizes Curriculares Nacionais Gerais da Educação Básica (BRASIL, 2013), oficializam esse desejo.

Em face das dificuldades normalmente já apresentadas pelos estudantes do EM no contato com os construtos da FC (GONÇAVES JR.; BARROSO, 2014), o ensino de FMC nesse nível de ensino - nos parece ser um desafio ainda maior, pois a maioria dos seus conceitos são não-intuitivos, diferentemente de boa parte dos conceitos da FC com os quais os alunos já estão, em teoria, familiarizados. Não obstante, parece haver hoje em dia um verdadeiro abismo entre a Física ensinada na sala de aula e a verificada no dia a dia dos estudantes. O que fazer, diante dessa situação? Procurar novas alternativas, em contrapartida ao ensino tradicional da Física, nos parece ser o melhor caminho.

$\mathrm{Na}$ tentativa de atenuar essa dicotomia, diversas propostas didáticas e metodologias de ensino surgiram ao longo dos anos. Por exemplo, Ricci, Ostermann e Prado (2007) apresentam um trabalho onde tentam estabelecer uma conexão entre assuntos clássicos e quânticos através do interferômetro de Mach-Zehnder. Na mesma linha, Pereira et al. (2012) utilizam uma simulação virtual do interferômetro de Mach-Zehnder para discutir os postulados da Mecânica Quântica (MQ). Silva e Assis (2012) propõem uma atividade experimental para abordar o efeito fotoelétrico de um modo contextualizado. Já Silva e Júnior (2014) partem de uma abordagem semiclássica a uma abordagem quântica na discussão do efeito Compton, sob uma perspectiva histórica. Essas propostas parecem ser uma boa maneira de diminuir a distância entre o clássico e o quântico, seja abordando diretamente os conceitos quânticos, seja tentando relacioná-los a conceitos clássicos.

Também é inegável, no Ensino de Física, a importância das atividades experimentais. Sejam elas realizadas por meios reais ou virtuais, em laboratórios de Física ou Informática, as 
atividades experimentais são capazes de potencializar o processo de ensino e aprendizagem nas diferentes áreas de conhecimento da Física. A falta de um laboratório especializado - ou de material para realizar os experimentos - não é justificativa para impedir a utilização de experimentos, pois cada vez mais surgem propostas que utilizam materiais de baixo custo como é o caso da borracha quântica. Tais afirmações podem ser corroboradas através de uma breve consulta à literatura (ver, por exemplo, NOGUEIRA et al., 2000; ARAUJO; ABIB, 2003; PIRES; VEIT, 2006; CARRASCOSA et al., 2008; ARAUJO; VEIT, 2011; PEREIRA; MOREIRA, 2017; PEREZ et al., 2018).

Não foi encontrada na literatura brasileira qualquer proposta educacional ou referência ao experimento da borracha quântica. Junior e Lunazzi (2015) apenas trazem um guia de como montar o experimento. Uma menção mais sofisticada surgiu através de Hillmer e Kwiat (2007), numa abordagem que apenas sugere a montagem do aparato experimental, sem, no entanto, fazer qualquer aprofundamento conceitual, epistemológico ou didático. Já propostas que têm por base o experimento da dupla-fenda - que serve de pano de fundo ao entendimento da borracha quântica - são mais comuns na literatura (ver, por exemplo, FERREIRA; SOUZA FILHO, 2016).

Este trabalho foi motivado por algumas questões que ainda estão em aberto no atual cenário da Pesquisa em Ensino de Física: como aproximar o Ensino de Física tradicional, marcado pelo tecnicismo e pelos extensos currículos baseados na FC, dos anseios e aspirações de nossos alunos, que estão atualmente cercados por tecnologias baseadas quase que exclusivamente na FMC? De que modo ensinar conceitos de MQ aos alunos do EM utilizando aparatos experimentais, uma vez que tais equipamentos envolvem alta tecnologia e são, em sua grande maioria, inacessíveis às nossas escolas? Como tratar de conceitos altamente abstratos e matematicamente complexos, como os da MQ, com alunos do EM, de forma que eles compreendam minimamente a essência de tais conceitos? Entendemos que a presente atividade pode trazer alguma luz a estas questões.

Isto posto, o presente trabalho pretende apresentar ao professor de Física do EM uma atividade experimental que lhe permita trabalhar conceitos que remetem aos princípios fundamentais da Mecânica Quântica, através do experimento da borracha quântica, indicando formas de montagem e exploração de suas possibilidades. Essa sugestão de atividade experimental foi extraída de uma proposta didática mais ampla (SLOVINSCKI, 2017), que envolve uma sequência didática estruturada em cinco encontros, dentro de um contexto histórico do desenvolvimento da Teoria Quântica, para a qual referendamos os leitores para mais detalhes. Apesar dessa proposta ser voltada à Educação Básica, entendemos que ela pode servir como subsídio à introdução do tema FMC aos alunos da Formação Inicial de Professores, bem como para reforçar tais conceitos, se aplicada à capacitação e à Formação Continuada de Professores. 


\section{Referenciais teóricos}

Trataremos, a seguir, dos referenciais teóricos que embasam a presente atividade experimental: os postulados da MQ e a transposição didática de Yves Chevallard (1991).

\section{II.1 Os postulados da Mecânica Quântica}

$\mathrm{Na} \mathrm{FC}$, o estado de um sistema físico qualquer num instante de tempo $t$ é dado pelo conjunto de coordenadas generalizadas $q_{i}(t)$ e seus respectivos momentos lineares $p_{i}(t)$. Desse modo, uma grandeza física $A\left(q_{i}, p_{i}, t\right)$ é determinada completamente quando conhecemos $q_{i}(t)$ e $p_{i}(t)$. Se é dado um conjunto $\left\{q_{i}\left(t_{0}\right), p_{i}\left(t_{0}\right)\right\}$ num instante $t_{0}$, podemos encontrar $\left\{q_{i}(t), p_{i}(t)\right\}$ através das equações de Hamilton:

$$
\frac{\partial H}{\partial q_{i}}=-\dot{p}_{i} \quad ; \quad \frac{\partial H}{\partial p_{i}}=\dot{q}_{i}
$$

Já a MQ é descrita, conceitual e matematicamente, através de postulados (COHENTANNOUDJI; DIU; LALOË, 1977), que podem ser resumidos, de uma maneira sucinta, da seguinte forma:

- $1^{\mathrm{o}}$ postulado: é descrição do estado de um sistema físico. Em um instante de tempo $t_{0}$, o estado de um sistema físico é definido por um ket $\left|\psi\left(t_{0}\right)\right\rangle$, que pertence ao espaço vetorial de estado $\varepsilon$ do sistema, conhecido como espaço de Hilbert $^{2}$, e onde cada vetor representa um possível estado. Como $\varepsilon$ é um espaço vetorial, ele admite o princípio da superposição, ou seja, a combinação linear de vetores de estado é também um vetor de estado.

- $2^{\mathrm{o}}$ postulado: a toda quantidade física mensurável $\alpha$ pode ser associado um operador $A$ atuando em $\varepsilon$, e este operador é um observável. Em outras palavras, um observável é algo que pode ser determinado. As quantidades físicas $\alpha$ são determinadas através da equação de autovalores para o observável (Equação 2), o que implica na quantização dos possíveis resultados da medida para espectros discretos. No caso de medições de posição, por exemplo, os valores possíveis são contínuos.

\footnotetext{
2 Tecnicamente, um espaço de Hilbert é um espaço com produto interno completo, e o conjunto de funções de quadrado integrável é apenas um exemplo de um espaço de Hilbert; na verdade, todo espaço vetorial com dimensão finita é trivialmente um espaço de Hilbert. Porém, sendo L2 a arena da MQ, é a que os físicos geralmente se referem quando usam o termo 'espaço de Hilbert'. A propósito, a palavra completo significa que qualquer sequência de funções de Cauchy no espaço de Hilbert converge para uma função que também está no espaço: não há 'lacunas', assim como o conjunto de todos os números reais não tem lacunas (em contrapartida, o espaço de todos os polinômios, por exemplo, como o conjunto de todos os números racionais, certamente tem lacunas). A completude de um espaço não tem nada a ver com a completude (mesma palavra, infelizmente) de um conjunto de funções, que é a propriedade pela qual qualquer outra função tem de poder ser expressa como uma combinação linear dela (GRIFFITHS, 2011).
} 


$$
A\left|u_{n}\right\rangle=a_{n}\left|u_{n}\right\rangle
$$

- $3^{\circ}$ postulado: o único resultado possível de ser observado em uma medida da quantidade física $\alpha$ é um dos autovalores do observável $A$.

- $4^{\circ}$ postulado: ao realizar a medida do observável $A$ em um sistema descrito pelo vetor $|\psi\rangle$ normalizado, a probabilidade de se encontrar o autovalor $a_{n}$, no caso (mais simples) de um espectro não degenerado, é

$$
P\left(a_{n}\right)=\left|\left\langle u_{n} \mid \psi\right\rangle\right|^{2} .
$$

- $5^{\circ}$ Postulado: também conhecido como redução do pacote de onda. Se a medida da quantidade física $\alpha \mathrm{em}$ um sistema físico no estado $|\psi\rangle$ fornecer o resultado $a_{n}$, então o estado do sistema imediatamente após a medida é a projeção normalizada de $|\psi\rangle$, ou seja

$$
|\psi\rangle \rightarrow e^{i \phi}\left|u_{n}\right\rangle=\frac{P_{n}|\psi\rangle}{\sqrt{\left\langle\psi\left|P_{n}\right| \psi\right\rangle}}
$$

Onde $P_{n}=\left|u_{n}\right\rangle\left\langle u_{n}\right|$. Expandindo convenientemente o estado $|\psi\rangle$ em termos dos autovetores do operador correspondente à variável que se mede, $|\psi\rangle=$ $\sum_{j} a_{j}\left|u_{j}\right\rangle$ e aplicando o operador projeção, teremos uma delta de Kronecker que vai "aniquilar" o somatório em $\mathrm{j}$ e deixar o $n$-ésimo termo $a_{n}\left|u_{n}\right\rangle$ no denominador.

- $6^{0}$ Postulado: a evolução temporal do vetor de estado $|\psi(t)\rangle$ é descrita pela equação de Schrödinger (Equação 5), onde $H(t)$ é o observável associado à energia total do sistema

$$
i \hbar \frac{d|\psi(t)\rangle}{d t}=H(t)|\psi(t)\rangle
$$

\section{II.2 A transposição didática}

Uma vez que os postulados da MQ são extremamente abstratos e carregados de um formalismo matemático que foge do domínio de nosso público-alvo, esta atividade experimental se utilizou dos conceitos da transposição didática de Yves Chevallard (1991), que trata da distância e das modificações sofridas por um saber entre a sua concepção e futura inserção na sala de aula, dentro dos diversos níveis de ensino. A análise de como se dá a transformação de um conceito dentro dos níveis dos saberes é o foco do trabalho de Chevallard (1991). Para ele, um conceito, quando transposto, passa por severas modificações, mas mantém semelhanças com a ideia original, apesar de adquirir significados próprios do ambiente escolar. Assim, Chevallard (1991) espera que os saberes, no contexto do ensino, não 
sejam meras simplificações dos saberes iniciais, mas sim novos saberes capazes de responder aos domínios da ciência e da sala de aula.

Chevallard (1991) classifica e descreve os saberes em três níveis básicos. O Saber Sábio é concebido sob os pilares da Ciência por pesquisadores e cientistas e publicado em periódicos de referência pertencentes à sociedade especializada daquela área de conhecimento, pois suas especificidades e particularidades são intrínsecas àquela comunidade. Já o Saber a Ensinar é o resultado da transposição de algum conceito do Saber Sábio para o ambiente escolar de algum nível do ensino, adquirindo um caráter didático e se materializando em forma de livros didáticos, por exemplo. Por fim, o Saber Ensinado, carregado de um didatismo próprio, que é a maneira como o Saber Sábio se apresenta aos estudantes na sala de aula, moldado pela dinâmica da sala de aula, onde ocorre nova transposição. $\mathrm{O}$ estudante dará um significado próprio àquele conceito.

De acordo com Chevallard (1991), o sucesso para a transposição de um conceito depende de uma série de fatores. O conceito deve ser consensual e relevante perante sua comunidade científica e a sociedade. Além disso, deve ser capaz de gerar exercícios e outras formas de avaliação e criar uma identidade própria dentro do ambiente escolar. Esse conjunto de características dá a dimensão do que é necessário para que um conhecimento seja transposto do Saber Sábio, passando pelo Saber a Ensinar, e desembocando no Saber Ensinado, de maneira eficaz. A MQ se encaixa parcialmente nessas condições. Sua relevância perante a sociedade e a comunidade da Física é indiscutível. No entanto, ela ainda não está inserida no contexto escolar, carecendo de uma maior atenção, principalmente de nós, docentes. Assim, esta atividade experimental se utilizou da ideia de transposição didática de Chevallard (1991) para tentar adaptar os postulados da MQ ao nível cognitivo dos estudantes do EM. Todavia, nossa intenção não é apenas reduzir as abstrações necessárias para se tratar do tema, mas sim transpor didaticamente os conceitos da MQ, do Saber Sábio ao Saber a Ensinar, utilizando expressões e vocabulário condizentes, de modo a torná-los acessíveis ao público-alvo deste trabalho.

\section{II.3 Os princípios fundamentais da Mecânica Quântica}

Por uma questão didática e metodológica, tendo em vista o público-alvo e levando em consideração um dos referenciais teóricos deste trabalho, - a transposição didática - os postulados da MQ foram substituídos pela expressão princípios fundamentais da $M Q$ e tratados de maneira qualitativa, uma vez que não se adequam ao nível intelectual dos alunos, tampouco à presente atividade, devido, principalmente, à sua complexa descrição matemática. Assim, sempre que tal expressão surgir, a partir desse ponto, devemos entender que faz referência aos postulados da $\mathrm{MQ}$.

O primeiro princípio abordado é a função de onda. Cada partícula ou objeto do universo pode ser descrita por uma função de onda, que é bem determinada em cada instante de tempo e ponto do espaço. A função de onda é obtida matematicamente, sendo ela uma 
solução da equação de Schrödinger, que foi formulada por Erwin Schrödinger em 1926. A equação de Schrödinger é o principal descritor da MQ, e equivale às equações de movimento da Mecânica Clássica (MC). Ela determina todas as propriedades observáveis de um sistema físico, no espaço e no tempo. Uma das representações possíveis da Equação de Schrödinger, na forma independente do tempo, é apresentada na Equação 6 (GRIFFITHS, 2011).

$$
-\frac{\hbar^{2}}{2 m} \frac{d^{2} \psi}{d x^{2}}+V \psi=E \psi
$$

A simples observação da Equação 6 já demonstra o caráter qualitativo desta atividade, uma vez que é uma equação diferencial, com partes real e imaginária, e não consta dos currículos do EM. Entretanto, sua interpretação é simplificada quando analisada sob o ponto de vista da conservação da energia, um dos pilares da Física, e que vale tanto para a MC quanto para a MQ. A primeira parcela, à esquerda da igualdade da Equação 6, diz respeito à energia cinética da partícula, e a segunda se refere à energia potencial. As parcelas, somadas, resultam na energia total do sistema, à direita da igualdade, do mesmo modo como ocorre na MC.

Mas se a função de onda é a solução matemática da Equação de Schrödinger, qual o significado físico dessa solução? De acordo com a interpretação de Copenhague (OSTERMANN; PRADO, 2005), a solução apresenta todos os possíveis estados permitidos daquele sistema físico, sendo este outro princípio fundamental. Assim, um estado quântico fornece uma distribuição de probabilidade para o valor de cada observável. O conhecimento do estado quântico e das regras para a evolução do sistema no tempo traz toda a informação sobre o comportamento do sistema. Um bom exemplo do conceito de estado são os níveis de energia possíveis para o átomo de hidrogênio, onde cada nível corresponde a uma energia bem determinada. Convém ressaltar que esses valores de energia podem ser obtidos experimentalmente e são solução da equação de Schrödinger para o átomo de hidrogênio. A Fig. 1 traz um diagrama dos níveis de energia do átomo de hidrogênio, com seus respectivos valores quantizados.

Se o conceito de estado não causa grande surpresa quanto ao seu significado, o próximo princípio fundamental pode causar. A solução da Equação de Schrödinger determina os possíveis estados observáveis de um sistema físico e não há um limite para a quantidade deles. Assim, enquanto não é feita nenhuma observação, em qual estado o sistema físico se encontra? Novamente, pela interpretação adotada, dizemos que o estado físico do sistema é indeterminado, onde não há uma energia definida para o mesmo. É a chamada superposição de estados e significa dizer que todos os estados coexistem ao mesmo tempo enquanto nenhum deles é observado.

Se todos os estados preditos pela função de onda estão coexistindo simultaneamente, existe uma maneira de saber qual estado será assumido pelo sistema físico estudado? A resposta é não - o estado somente será conhecido quando for observado. Pode, contudo, haver um estado preferencial que pode ter maior possibilidade de ser observado em relação a outro. 
Esse princípio é chamado de probabilidade e surge de uma operação matemática discutida anteriormente nos postulados. Esse recurso matemático possibilita que se conheça, numericamente, qual a probabilidade de se observar cada estado predito pela função de onda.

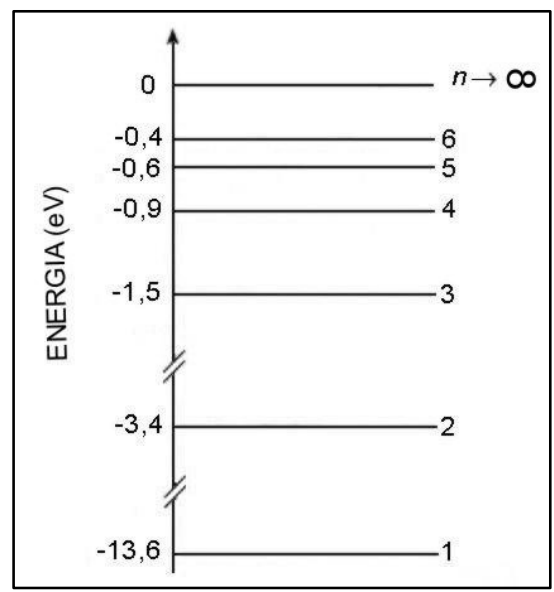

Fig. 1 - Diagrama dos níveis de energia do átomo de hidrogênio. FONTE: $<$ http://www.ebah.com.br/content/ABAAAfwGUAA/teoria-quantica-atomo-hidrogenio>.

Mas o que significa, fisicamente, observar algum estado? De acordo com a interpretação adotada, o ato de observar algum dos estados possíveis nada mais é do que determinar o estado o sistema físico em questão. A determinação do estado é outro princípio da MQ e é nesse ponto, principalmente, que as diversas interpretações quanto à teoria começam a divergir. Devemos lembrar, quando da abordagem do princípio da incerteza de Heisenberg, que a simples observação de sistemas quânticos acaba por modificá-los, uma vez que na interação da radiação com a matéria há transferência de energia através do momentum linear dos fótons. Quando se trata da $\mathrm{MQ}$, o simples ato de determinar qualquer propriedade observável do sistema físico em questão acaba por alterar tal sistema. Assim, a função de onda - descrita inicialmente por $n$-estados possíveis - se altera, passando dos possíveis $n$ estados ao único estado observado. Esse princípio é chamado de colapso da função de onda e é um dos pontos mais controversos da Interpretação de Copenhague. Interpretações paralelas, como a Interpretação dos Universos Múltiplos ou a Interpretação da Onda Piloto (BETZ, 2014) discordam da Interpretação de Copenhague ao afirmar que a função de onda não colapsa, mas evolui conforme cada teoria.

De um modo geral, os princípios fundamentais da MQ podem ser descritos da seguinte maneira: a toda partícula (ou sistema físico qualquer) cabe uma função de onda, que descreve como se dará sua evolução no tempo e no espaço. Essa função de onda é a solução da Equação de Schrödinger aplicada à partícula e estabelece todos os estados observáveis. Enquanto nenhuma observação é realizada, a partícula encontra-se numa superposição de estados, isto é, todos os estados possíveis estão coexistindo simultaneamente. A função de onda também estabelece a probabilidade de cada estado ser observado. Realizada a 
determinação do estado, ocorre o colapso da função de onda, ou seja, ela colapsa de $n$-estados possíveis para o estado observado. A Equação 7 traz um exemplo simplificado de uma função de onda. A função $\Psi$ apresenta três estados possíveis $\left(\psi_{A}, \psi_{B}, \psi_{C}\right)$ e a cada estado cabe sua respectiva probabilidade de observação $(x, y, z)$ (o leitor mais atento perceberá que a notação bracket não será utilizada aqui).

$$
\Psi=x \psi_{A}+y \psi_{B}+z \psi_{C}
$$

Suponhamos que tenhamos determinado o estado do sistema físico e o estado observado foi o estado $\psi_{A}$. Ocorre então o colapso da função de onda, ou seja, a função que descrevia três estados possíveis, com as respectivas probabilidades de observação de cada estado, passa a conter apenas o estado observado, como mostra a Equação 8.

$$
\Psi=\psi_{A}
$$

\section{O experimento da borracha quântica}

A montagem do experimento da borracha quântica é relativamente simples, mas requer uma preparação inicial de seus componentes. Sugerimos que a montagem e os testes com o material sejam feitos com antecedência, para que não ocorram imprevistos durante a apresentação e discussão do experimento, uma vez que os polarizadores podem não funcionar da maneira esperada. O experimento da borracha quântica foi preparado seguindo as instruções de Junior e Lunazzi (2015), cujo arranjo experimental por nós produzido é trazido na Fig. 2, numa visão geral, e na Fig. 3, numa visão mais detalhada. Os polarizadores foram obtidos a partir de mostradores digitais de calculadoras velhas, conforme sugerido pelos autores.

Apesar do experimento da borracha quântica ser completamente descrito pela FC (uma vez que não conta com nenhum aparato tecnológico, tampouco consegue manipular fótons individualmente), seus resultados experimentais clássicos podem servir de análogo para o entendimento de fenômenos quânticos. Um esquema simplificado do experimento pode ser visualizado na Fig. 4, sendo o mesmo composto por quatro itens: uma fonte emissora de luz polarizada (laser, cujo modelo utilizado em nosso experimento é usado em laboratórios didáticos de Física, com comprimento de onda de $635 \mathrm{~nm}$, mas poderia ser de uma caneta ou apontador do tipo laser-pointer, de diferentes comprimentos de onda, facilmente encontrado no comércio popular); o identificador de caminho, um arranjo de dois filmes polarizadores fixos, orientados perpendicularmente entre si, com um arame fino fixado na junção deles, a fim de servir de obstáculo ao feixe de luz que passará por ele (o leitor perceberá, ao observar a figura, que o sentido de polarização dos filmes é dado pelas linhas horizontais e verticais do modelo esquemático); o analisador - um outro filme polarizador, agora móvel, cuja orientação pode ser ajustada em relação ao identificador de caminho (disposto, na figura, de modo a formar um ângulo de $45^{\circ}$ em relação aos polarizadores do identificador de caminho); 


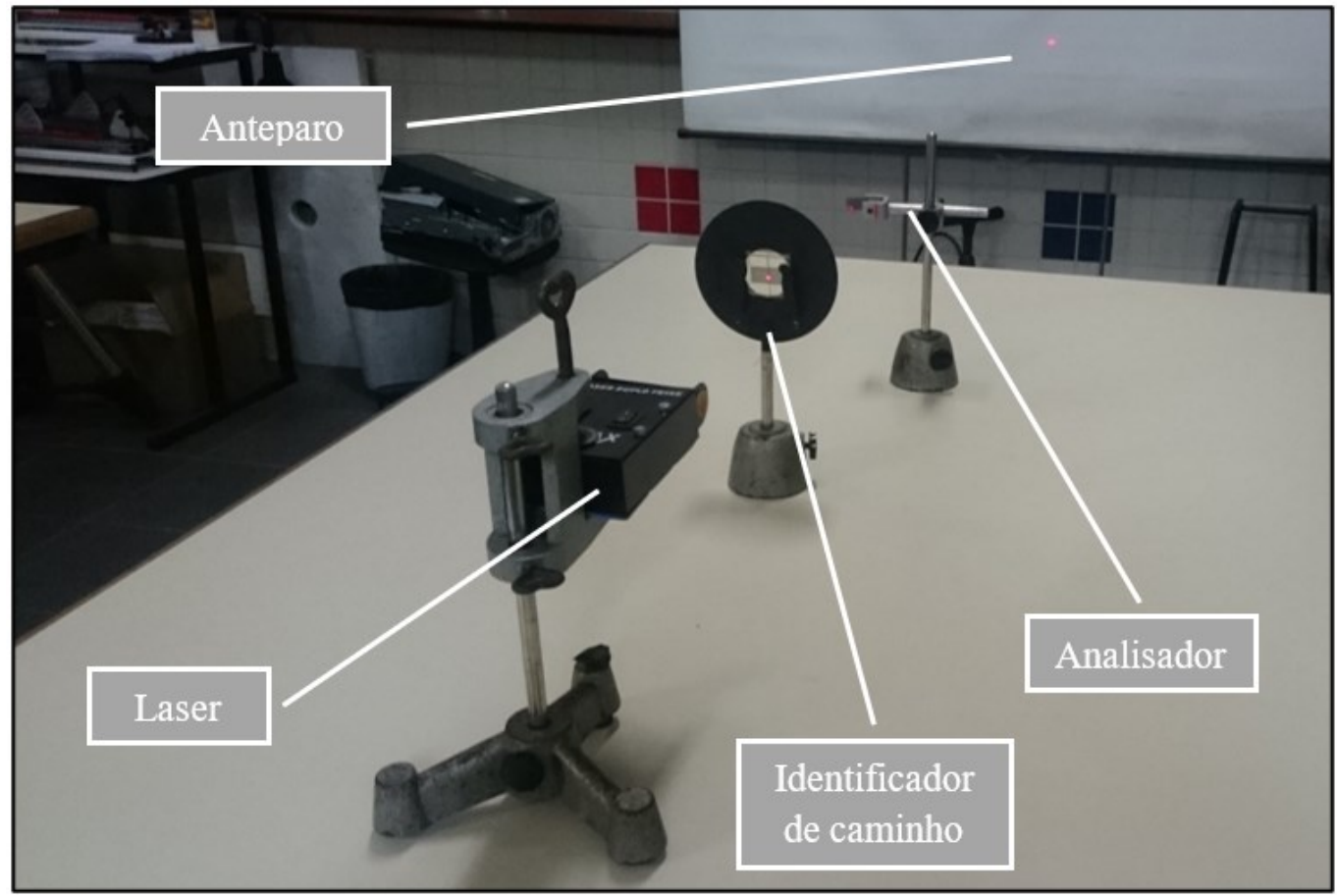

Fig. 2 - Visão geral do experimento da borracha quântica. FONTE: os autores.

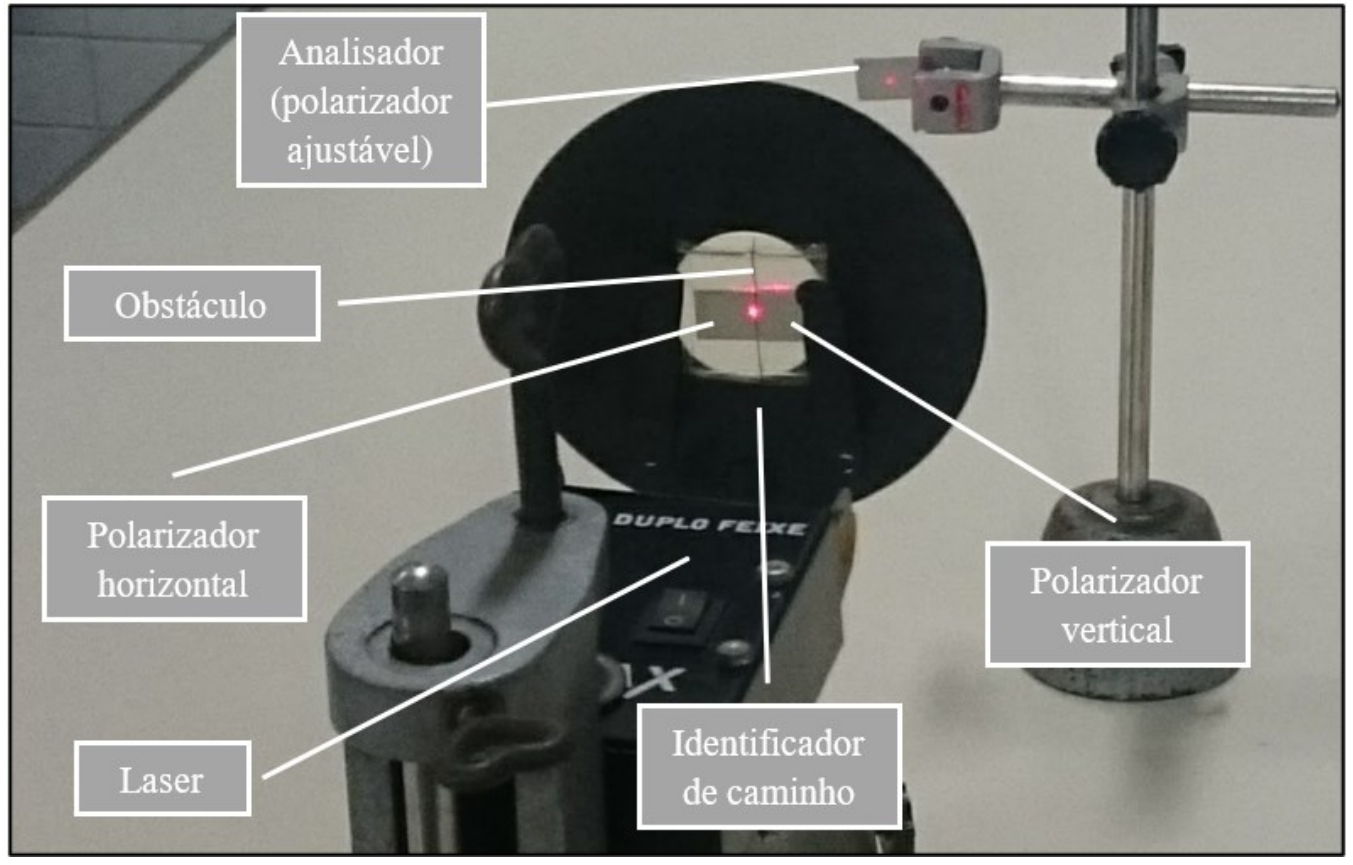

Fig. 3 - Visão detalhada da fonte, identificador de caminho e analisador. FONTE: os autores.

e um anteparo, onde se formarão padrões que serão identificados como sendo características de onda (quando surgir uma padrão de franjas de interferência) ou partícula (quando o padrão de franjas de interferência for perdido e surgir apenas um "borrão"), de acordo com a 
configuração do experimento. Esses padrões formados no anteparo serão melhor observados se o ambiente onde ocorrer o experimento estiver escurecido.

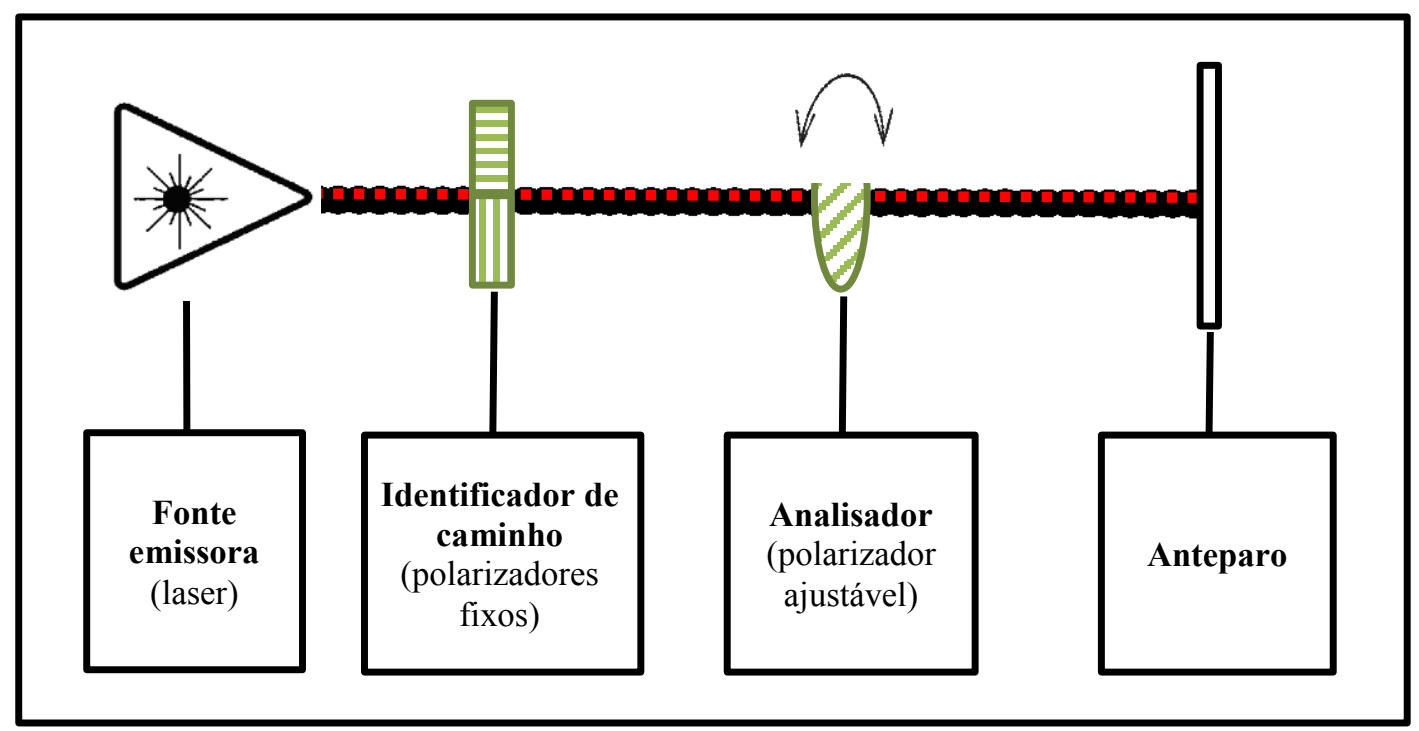

Fig. 4 - Representação esquemática de uma borracha quântica (forma completa). FONTE: os autores.

Antes da apresentação do experimento da borracha quântica, cabe a discussão de uma pequena curiosidade sobre o mesmo, que diz respeito à tradução do termo borracha quântica (do inglês quantum eraser). Talvez o termo que melhor traduziria a característica do experimento seria apagador quântico, uma vez que, como será mostrado, o analisador tem a função de apagar qualquer informação obtida sobre os fótons que passaram pelo identificador de caminho. A palavra borracha pode causar certa confusão ao leitor desavisado, que pode interpretá-la como sendo uma borracha escolar (que estaria correto) ou uma liga de borracha qualquer, utilizada para as mais diversas finalidades, e que nada tem a ver com o experimento. Todavia, o termo borracha quântica continuará a ser empregado ao longo deste trabalho, uma vez que o termo já está consagrado na literatura lusófona.

Convém ressaltar, ainda, que todas as imagens obtidas experimentalmente apresentaram um padrão que se assemelha às que serviram de base para a comparação, obtidas por Hillmer e Kwiat (2007). Entretanto, para o nosso experimento, montado no laboratório, foram utilizados materiais alternativos e de baixo custo - principalmente os filtros polarizadores, figuras principais do experimento - provenientes de telas LCD de calculadoras e mostradores digitais. A baixa qualidade desses materiais, aliada aos métodos de obtenção deles, fizeram com que as imagens resultantes dos fenômenos ficassem um tanto quanto visualmente poluídas. 


\section{Resultados experimentais}

O primeiro fenômeno mostrado é a interferência com ondas luminosas. Para isso, o experimento foi montado inicialmente apenas com a fonte emissora (laser) passando por um pequeno obstáculo (um arame fino ou até mesmo um fio de cabelo) e o anteparo, como mostra a Fig. 5. Essa configuração serve de análogo ao experimento da dupla fenda de Young . A explicação clássica do fenômeno diz que a onda incidente sobre o obstáculo se divide em outras duas ao ultrapassá-lo e essas acabam por interferir entre si formando no anteparo um padrão de franjas de interferência. No entanto, sob a ótica da $\mathrm{MQ}$, podemos entender que cada fóton que passa pelo obstáculo interfere consigo mesmo (CARUSO; OGURI, 2016) - ou então que os estados possíveis estão superpostos - motivo pelo qual aparecem as franjas de interferência. Os alunos devem ser alertados que a fonte emite vários fótons simultaneamente, não sendo possível, com este experimento, realizar a emissão de um único fóton por vez.

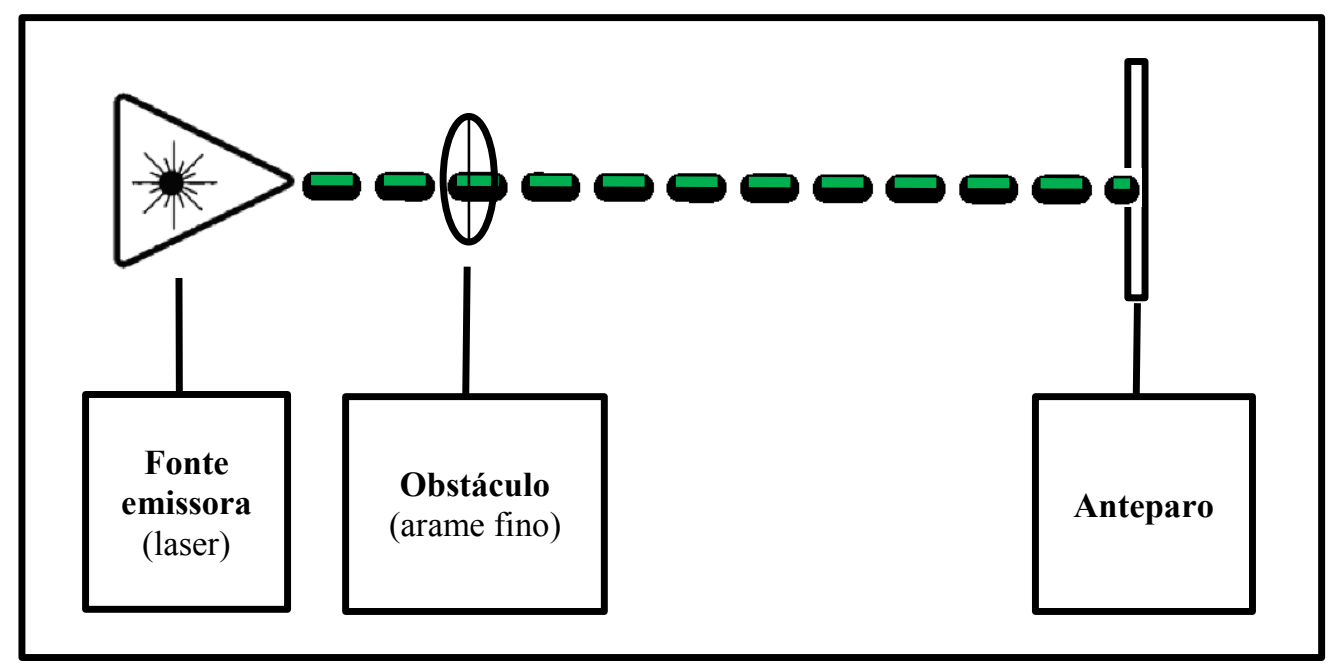

Fig. 5 - Representação esquemática de um experimento de interferência de ondas luminosas. FONTE: os autores.

Neste ponto, identificamos os seguintes princípios fundamentais da MQ: os estados possíveis daquele sistema físico (fóton passando pela direita ou esquerda do obstáculo), a

\footnotetext{
${ }^{3} \mathrm{O}$ experimento da dupla fenda proposto por Thomas Young exibe uma configuração em que uma onda plana originalmente incide sobre um obstáculo (muito maior que o comprimento da onda) que possui duas fendas (com tamanho próximo ao do comprimento da onda), adequadamente distantes entre si, e que funcionam como fontes pontuais da onda plana original (mesma frequência). Essas duas novas frentes de ondas interferem entre si, dando origem ao fenômeno da interferência. Já na configuração proposta pelo experimento, a onda plana original encontra um obstáculo (arame ou fio de cabelo) com tamanho próximo ao do comprimento da onda. Ao contornar o obstáculo, cada lado da onda original se comporta como uma nova frente de onda, e essas frentes acabam por também interferir entre si.

4 Processos físicos que envolvem a emissão/absorção de um único fóton ou elétron são bastante explorados atualmente na área de informação quântica, como os protocolos de criptografia, além de possuírem outras aplicações como o estudo do sequenciamento de DNA e a tomografia por emissão de pósitrons.
} 
forma da função de onda associada, a superposição dos estados possíveis e a probabilidade de se conhecer o lado pelo qual os fótons estão passando. Ressaltamos, novamente, que como o experimento não permite a emissão de uma única partícula por vez, temos vários fótons passando por ambos os lados do obstáculo ao mesmo tempo e, assim, o princípio da probabilidade não se aplica tão bem nessa situação. Nessa configuração, a imagem obtida no anteparo corresponde a uma figura de interferência, como já discutido anteriormente. Os resultados alcançados em nosso laboratório encontram-se nas imagens superiores da Fig. 6. A imagem (6a) traz o resultado experimental obtido por Hillmer e Kwiat (2007), que serviu de base a este trabalho. A imagem (6b) traz o resultado experimental obtido no laboratório, durante o experimento. Para fins de comparação, as figuras superiores (6a e 6b) são imagens retiradas diretamente dos anteparos, sendo que a obtida em nosso laboratório não possui nenhum tipo de tratamento especial. Já as figuras inferiores, (6c) e (6d), são as imagens (6a) e (6b) submetidas a um tratamento especial (filtro), cuja finalidade foi destacar as extremidades da figura, diminuindo o excesso de luz e dando ênfase às bordas da imagem, tudo no intuito de facilitar a percepção e verificar se ali podia ser identificado algum padrão conhecido ou esperado. Esse mesmo tratamento foi aplicado em todos os resultados que são apresentados na sequência. Dessa forma, percebemos que ambos os experimentos conseguiram, de alguma maneira, obter uma figura de interferência - como era esperado - já que o experimento simulou, nessa configuração, um obstáculo do tipo dupla-fenda.

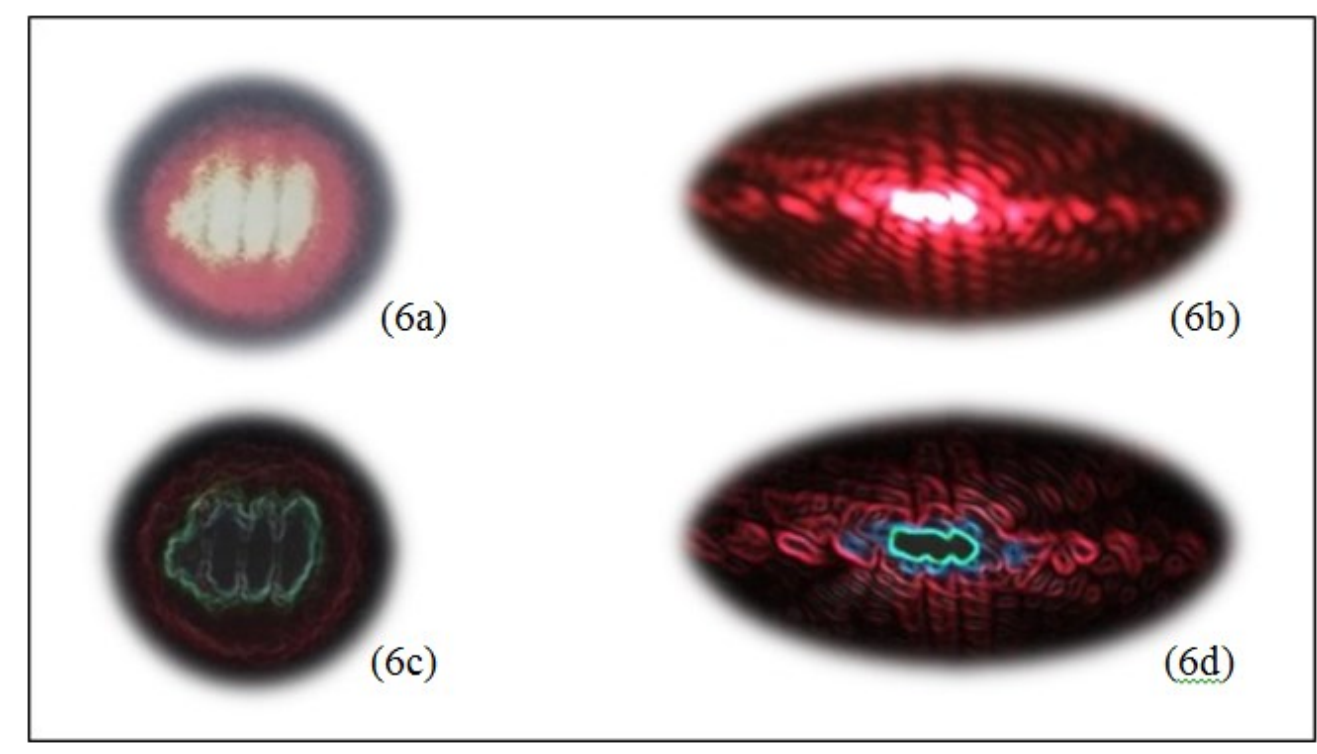

Fig. 6 - Resultados experimentais obtidos com o experimento de interferência de ondas luminosas. FONTE: imagem (6a): Hillmer e Kwiat (2007); imagens (6b), (6c) e (6d): os autores. 
A seguir, o arame foi substituído pelo identificador de caminho, conforme retratado na Fig. 7. Quando o experimento adquire essa configuração, o identificador de caminho polariza os fótons que passam pela esquerda e pela direita do arame, de acordo com a orientação dos polarizadores (horizontal ou vertical). Com isso, ocorre que estamos tentando obter alguma informação a respeito dos fótons, o que equivale, dentro dos princípios estudados, a determinar o estado em que se encontra o sistema em questão, devido à capacidade de seleção dos fótons pelo identificador de caminho. Isso, segundo a interpretação de Copenhague, causará o colapso da função de onda, evento que pode ser verificado pela destruição das franjas de interferência projetadas no anteparo, uma vez que o identificador de caminho foi capaz de informar o trajeto que cada fóton seguiu, impedindo que a interferência ocorra.

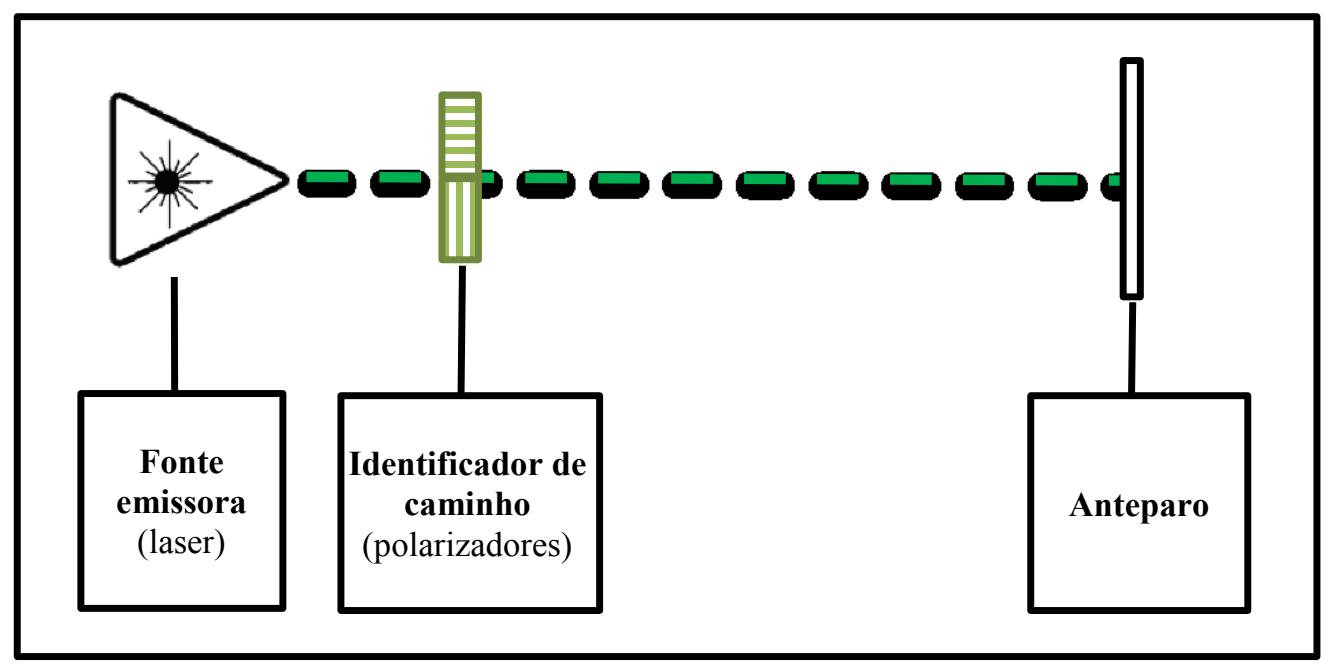

Fig.7 - Representação esquemática do experimento da borracha quântica, quando montada apenas com o identificador de caminho. FONTE: os autores.

De fato, o que se obtém é um borrão (ou a perda das franjas de interferência), como mostra a imagem (8b) da Fig. 8, a qual se assemelha à imagem (8a) da mesma figura. Podemos perceber com bastante nitidez, inclusive com o auxílio das figuras (8c) e (8d), tratadas com o filtro, que as figuras de interferência da Fig. 6 não mais existem, o que corrobora o conceito do colapso da função de onda.

A seguir, foi inserido no experimento o segundo polarizador (analisador), que deixou o experimento da borracha quântica completo, conforme mostrado na Fig. 4. O analisador foi colocado, inicialmente, na orientação horizontal, o que obrigatoriamente o deixou perpendicularmente orientado em relação a um dos polarizadores do identificador de caminho, e paralelamente orientado em relação ao outro. Com isso, metade dos fótons foram bloqueados pelo analisador, enquanto a outra metade não foi afetada. O resultado desse arranjo experimental foi um acúmulo de fótons em um dos lados do borrão, que equivale à 
orientação de polarização do analisador. Mesmo que essa parte do experimento não tenha abrangido nenhum princípio estudado, pode ser interessante mostrar aos alunos como se dá a manipulação de fótons em um experimento que utiliza filtros polarizadores.

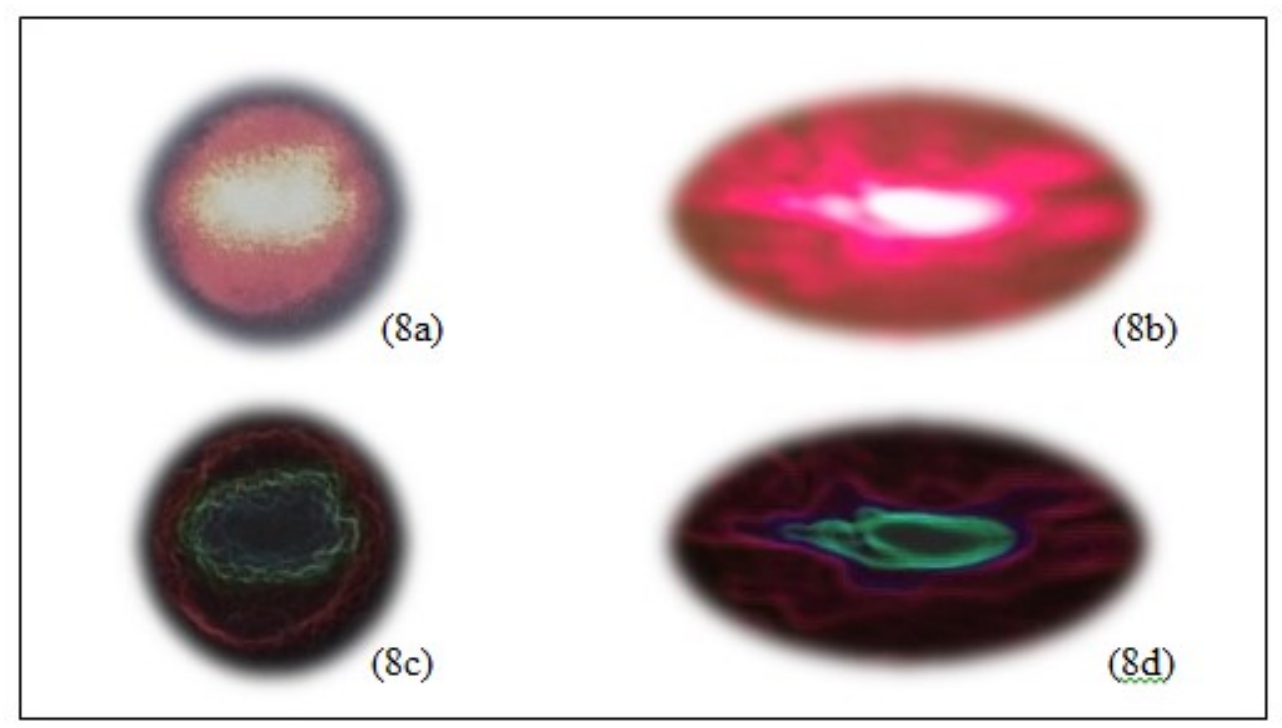

Fig. 8 - Resultados experimentais obtidos com a borracha quântica, quando montada apenas com o identificador de caminho. FONTE: imagem (8a): Hillmer e Kwiat (2007); imagens (8b), (8c) e (8d): os autores.

A Fig. 9, a exemplo das demais, traz na imagem (9b) o resultado experimental obtido no laboratório, em comparação com os resultados alcançados por Hillmer e Kwiat (2007), que utilizaram o analisador nas orientações horizontal e vertical, visto na imagem (9a). Mais uma vez, foi possível perceber que houve semelhança em relação aos padrões dos dois experimentos. Verificamos, também, que o resultado experimental obtido em sala de aula estava com os fótons mais concentrados à direita, quando comparado com o resultado exposto imagem (8b) da Fig. 8, mostrando a capacidade de manipulação dos fótons.

Pelo apresentado até aqui, o experimento da borracha quântica consegue tratar de todos os princípios fundamentais da MQ propostos. Se montado conforme a Fig. 5, temos um sistema físico que pode ser descrito por uma função de onda, de onde podemos calcular a probabilidade de se encontrar um dos dois estados possíveis. Apesar de um imenso número de fótons estar passando pelos dois lados do arame ao mesmo tempo, quanticamente podemos entender que cada fóton interfere consigo mesmo, formando uma figura de interferência sobre o anteparo. Além disso, não se pode afirmar por qual lado do arame o fóton está passando, e com isto temos a superposição de estados. Ao montarmos o experimento conforme a Fig. 7, temos uma tentativa de obter informações a respeito do fóton, o que equivale a determinar $\mathbf{0}$ estado do sistema, tendo como consequência o colapso da função de onda, e como resultado, a perda da figura de interferência no anteparo. As informações decorrentes da 
tentativa de observação dos fótons se materializam nas Fig. 8 e 9, com uma maior concentração deles ocorrendo à esquerda ou à direita da posição inicial, de acordo com o posicionamento do analisador.

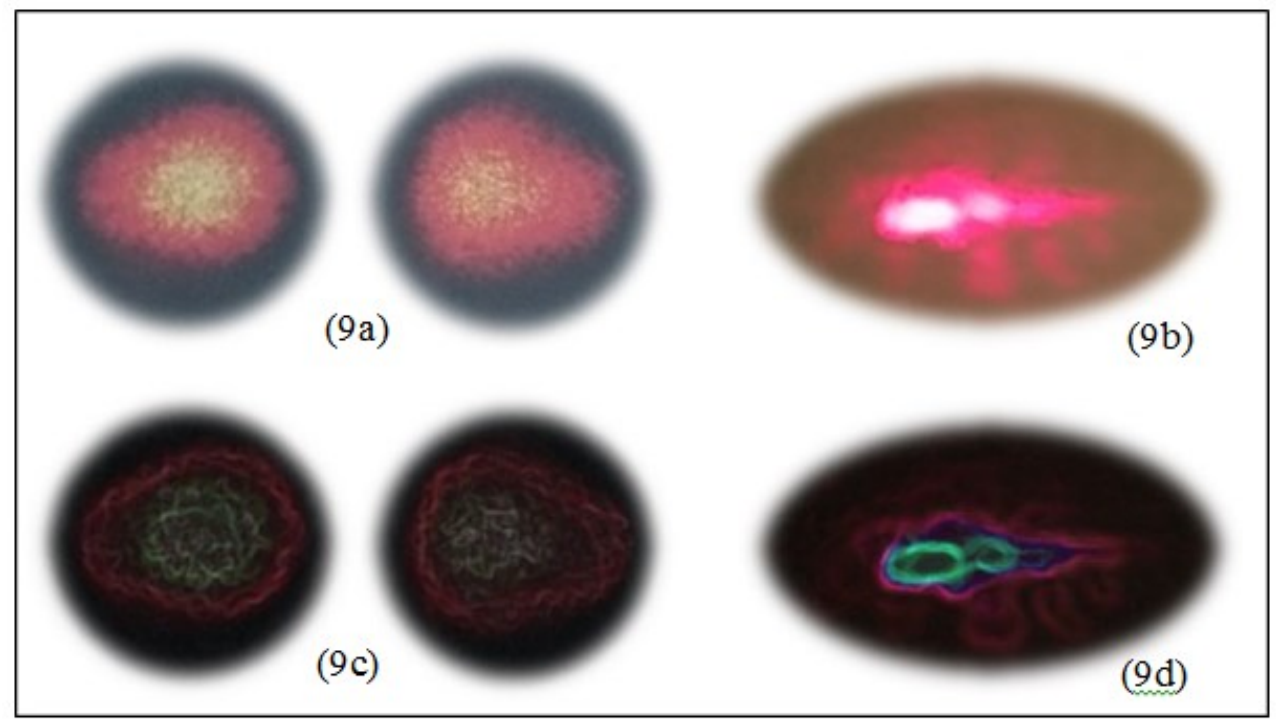

Fig. 9 - Resultados experimentais obtidos com o experimento da borracha quântica (seleção de fótons). FONTE: imagem (9a): Hillmer e Kwiat (2007); imagens (9b), (9c) e (9d): os autores.

A teoria quântica aqui estudada, de acordo com a Interpretação de Copenhague, prevê que, uma vez colapsada, a função de onda não pode mais retomar sua forma original naquele determinado sistema físico, ou seja, a informação perdida não pode mais ser recuperada. Mas, de alguma forma, seria possível readquirir essa informação? A grande surpresa do experimento da borracha quântica é que o padrão das franjas de interferência, perdido por ocasião da atuação do identificador de caminho, pode ser recuperado. Para isso, basta girar o analisador de $45^{\circ}$ em qualquer sentido, horário ou anti-horário, e temos o restabelecimento do padrão de interferência. Isso acontece porque o analisador consegue "apagar" a informação obtida através do identificador de caminho, sobre por qual lado do arame o fóton passou, permitindo ao mesmo um retorno a um estado tal como o original. Assim, devido a essa nova configuração do experimento, cada fóton com polarização vertical ou horizontal tem a mesma chance de ter passado por um dos lados do arame, uma vez que nada mais se sabe a seu respeito. O resultado prático disso é o reaparecimento das franjas de interferência, o que pode ser verificado na imagem (10b) da Fig. 10. Aqui, percebemos novamente que o resultado experimental obtido em sala de aula se aproxima da imagem (10a) da mesma figura.

Ao atravessar o analisador, os fótons perdem a polarização adquirida quando da passagem pelo identificador de caminho e adquirem uma nova polarização. Ou seja, cada 
fóton com polarização horizontal ou vertical agora tem $50 \%$ de chance de chegar ao anteparo por quaisquer dos caminhos que seguiu, o que resulta na impossibilidade de determinar sua trajetória. Assim, é como se cada fóton atravessasse o obstáculo pelos dois lados simultaneamente, interferindo consigo mesmo, e reestabelecendo as franjas de interferência.

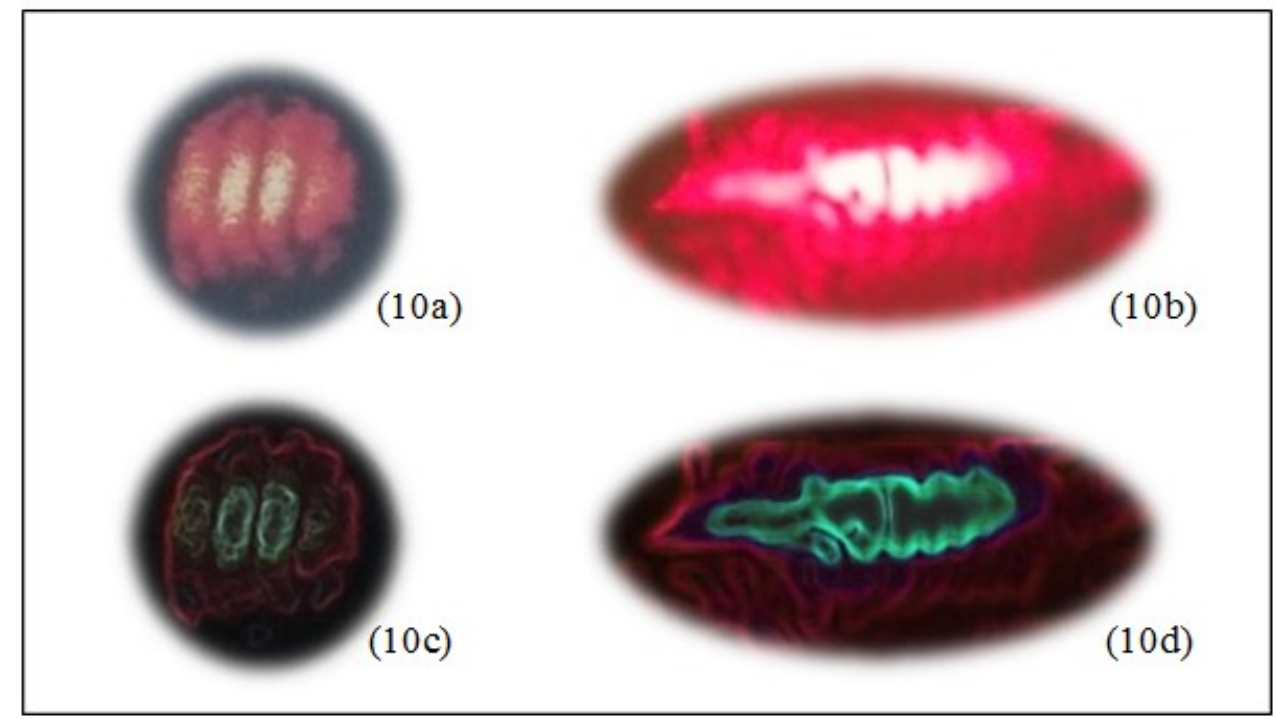

Fig. 10 - Resultados experimentais obtidos com o experimento da borracha quântica (reaparecimento das franjas de interferência). FONTE: imagem (10a): Hillmer e Kwiat (2007); imagens (10b), (10c) e (10d): os autores.

\section{Considerações finais}

A presente atividade experimental baseou-se em conhecimentos da FC para introduzir conceitos de MQ a estudantes do EM por meio de um experimento real. Estratégia semelhante foi empregada por Ricci, Ostermann e Prado (2007), que utilizaram uma simulação computacional baseada na Mecânica Ondulatória Clássica como porta de entrada para o ensino da MQ em cursos introdutórios de FMC no Ensino Superior.

Pelo que foi exposto no referencial teórico e discutido nos resultados experimentais, podemos perceber que é fundamental que os conhecimentos referentes ao Saber Sábio da MQ sejam didaticamente transpostos e formem um Saber a Ensinar compatível com o nível cognitivo dos alunos do EM. Esse processo de transposição didática deve levar em conta as diferentes formas de aprender dos estudantes, e não podemos conceber que todos eles irão aprender da mesma maneira e na mesma velocidade - daí a importância da abordagem qualitativa dos conceitos de MQ aqui trazidos. Assim, entendemos que este trabalho oportuniza uma maior aproximação entre esses conhecimentos e os estudantes do EM, atendendo às questões que motivaram a presente atividade. 
Apesar de permitir a abordagem e discussão dos princípios fundamentais da MQ sugeridos, o experimento da borracha quântica é explicado pela Mecânica Ondulatória Clássica - é a chamada restauração clássica do padrão de interferência. Como ressaltado anteriormente, um experimento que envolva manipulação individual de fótons ou partículas só é possível com equipamentos encontrados em avançados laboratórios de pesquisa, o que não é caso de nenhum laboratório escolar do Brasil. Todavia, entendemos que o experimento clássico aqui proposto pode servir como análogo quântico e suprir essa lacuna do ensino.

Os resultados experimentais alcançados foram considerados bastante próximos do previsto pela teoria, a despeito dos filtros polarizadores usados. Esse fato reforça as diversas propostas que incentivam o uso de materiais de baixo custo, que se mostram uma alternativa bastante viável à falta de recursos observada em grande parte das escolas brasileiras.

\section{Referências}

ARAUJO, M. S. T.; ABIB, M. L. V. S. Atividades experimentais no ensino de física: diferentes enfoques, diferentes finalidades. Revista Brasileira de Ensino de Física, São Paulo, v. 25, n. 2, p. 176-194, jun. 2003.

ARAUJO, I.; VEIT, E. Uma revisão da literatura sobre estudos relativos a tecnologias computacionais no Ensino de Física. Revista Brasileira de Pesquisa em Educação em Ciências, v. 4, n. 3, mar. 2011.

ARONS, A. B. A guide to introductory physics teaching. New York: John Wiley, 1990.

ARRUDA, S. M.; VILLANI, A. Sobre as origens da relatividade especial: relações entre quanta e relatividade em 1905. Caderno Brasileiro de Ensino de Física, Florianópolis, v. 13, n. 1, p. 32-47, abr. 1996.

BETZ, M. E. M. Elementos de mecânica quântica da partícula na interpretação da onda piloto. Revista Brasileira de Ensino de Física, São Paulo, v. 36, n. 4, 4310, dez. 2014.

BRASIL. Ministério da Educação. Diretrizes Curriculares Nacionais Gerais da Educação Básica. Brasília: MEC/SEB, 2013.

BRASIL. Ministério da Educação. Orientações Curriculares para o Ensino Médio. Brasília: MEC/SEB, 2006.

BRASIL. Ministério da Educação. Orientações Educacionais Complementares aos Parâmetros Curriculares Nacionais. Brasília: MEC/SEB, 2002. 
BRASIL. Ministério da Educação. Parâmetros Curriculares Nacionais: Ensino Médio. Brasília: MEC/SEB, 1999.

BROCKINGTON, G.; PIETROCOLA, M. Serão as regras da transposição didática aplicáveis aos conceitos de física moderna? Investigações em Ensino de Ciências, Porto Alegre, v. 10, n. 3, p. 387-404, dez. 2005.

CARRASCOSA, J. et al. Papel de la actividad experimental en la educación científica. Caderno Brasileiro de Ensino de Física, Florianópolis, v. 23, n. 2, p. 157-181, set. 2008.

CARUSO, F.; OGURI, V. A estranha teoria quântica da luz. Ciência e Sociedade, Rio de Janeiro, v. 4, n. 2, p. 12-17, 2016.

CHEVALLARD, Y. La transposición didáctica: del saber sabio al saber enseñado. Buenos Aires: La Pensée Sauvage, 1991.

COHEN-TANNOUDJ, C.; DIU, B.; LALOË, F. Quantum mechanics. New York: Wiley, 1977.

FELDENS, B.; DIAS, P. M. C.; SANTOS, W. M. S. E assim se fez o quantum... Revista Brasileira de Ensino de Física, São Paulo, v. 32, n. 2, 2602, Apr./June 2010.

FERREIRA, D. C.; SOUZA FILHO, M. P. O experimento virtual da dupla fenda ao nível de ensino médio (Parte I): uma análise clássica do comportamento corpuscular e ondulatório, e o desenvolvimento de um software computacional. Caderno Brasileiro de Ensino de Física, Florianópolis, v. 33, n. 2, p. 697-716, ago. 2016.

FISCHLER, H.; LICHTFELDT, M. Modern physics and student's conceptions. International Journal of Science Education, London, v. 14, n. 2, p. 181-190, Apr./June 1992.

GIL, D. P.; SENENT, F.; SOLBES, J. Análisis crítico de la introducción de la física moderna en la enseñanza media. Revista de Enseñanza de la Física, Rosario, v. 2, n. 1, p. 16-21, abr. 1988.

GONÇALVES JR., W. P.; BARROSO, M. F. As questões de física e o desempenho dos estudantes no ENEM. Revista Brasileira de Ensino de Física, São Paulo, v. 36, n. 1, 1402, fev. 2014. 
GRECA, I. M.; MOREIRA, M. A.; HERSCOVITZ, V. E. Uma proposta para o ensino de mecânica quântica. Revista Brasileira de Ensino de Física, São Paulo, v. 23, n. 4, p. 444457, dez. 2001.

GRIFFITHS, D. J. Introduction of quantum mechanics. New Jersey: Prentice Hall, 2011.

HILLMER, R.; KWIAT, P. A do-it-yourself quantum eraser. Scientific American, New York, v. 296, n. 5, p. 72-77, May 2007.

JUNIOR, J. H. F.; LUNAZZI, J. J. Construção de uma borracha quântica. Disponível em: $<$ http://www.ifi.unicamp.br/ lunazzi/F530_F590_F690_F809_F895/F809/F809_sem2_2007/J orgeH-MonicaRF.pdf>. Acesso em: 15 jun. 2015.

MOZENA, E. R.; OSTERMANN, F. Sobre a Base Nacional Comum Curricular (BNCC) e o Ensino de Física. Caderno Brasileiro de Ensino de Física, v. 33, n. 2, p. 327-332, ago. 2016.

NOGUEIRA, J. S. et al. Utilização do Computador como Instrumento de Ensino: Uma Perspectiva de Aprendizagem Significativa. Revista Brasileira de Ensino de Física, São Paulo, v. 22, n. 4, p. 517-522, dez. 2000.

OSTERMANN, F.; MOREIRA, M. A. Uma revisão bibliográfica sobre a área de pesquisa “física moderna e contemporânea no ensino médio". Investigações em Ensino de Ciências, Porto Alegre, v. 5, n. 1, p. 23-48, jan. 2000.

OTERMANN, F.; PRADO, S. D. Interpretações da mecânica quântica em um interferômetro virtual de Mach-Zehnder. Revista Brasileira de Ensino de Física, São Paulo, v. 27, n. 2, p. 193-203, jun. 2005.

PEREIRA, A. P. et al. Uma abordagem conceitual e fenomenológica dos postulados da física quântica. Caderno Brasileiro de Ensino de Física, Florianópolis, v. 29, n. Especial 2, p. 831-863, out. 2012.

PEREIRA, M. V.; MOREIRA, M. C. A. Atividades prático-experimentais no ensino de Física. Caderno Brasileiro de Ensino de Física, Florianópolis, v. 34, n. 1, p. 265-277, maio 2017.

PEREZ, S. et al. O estudo do movimento browniano com material de baixo custo. Revista Brasileira de Ensino de Física, São Paulo, v. 40, n. 1, e1503, 2018. 
PIRES, M. A.; VEIT, E. A. Tecnologias de Informação e Comunicação para ampliar e motivar o aprendizado de Física no Ensino Médio. Revista Brasileira de Ensino de Física, São Paulo, v. 28, n. 2, p. 241-248, jun. 2006.

RICCI, T. F.; OSTERMANN, F.; PRADO, S. D. O tratamento clássico do interferômetro de Mach-Zehnder: uma releitura mais moderna do experimento da fenda dupla na introdução da física quântica. Revista Brasileira de Ensino de Física, São Paulo, v. 29, n. 1, p. 79-88, mar. 2007.

SILVA, I.; JUNIOR, O. F. A descoberta do efeito Compton: de uma abordagem semiclássica a uma abordagem quântica. Revista Brasileira de Ensino de Física, São Paulo, v. 36, n. 1, p. 1601, fev. 2014.

SILVA, L. F.; ASSIS, A. Física Moderna no Ensino Médio: um experimento para abordar o efeito fotoelétrico. Caderno Brasileiro de Ensino de Física, Florianópolis, v. 29, n. 2, p. 313-324, ago. 2012.

SLOVINSCKI, L. O experimento da borracha quântica: uso de analogias para o entendimento do quântico pelo clássico. 2017. Dissertação (Mestrado Profissional em Ensino de Física) - UFRGS, Porto Alegre.

TERRAZZAN, E. A. A inserção da Física Moderna e Contemporânea no Ensino de Física na Escola de $2^{\circ}$ grau. Caderno Catarinense de Ensino de Física, Florianópolis, v. 9, n. 3, p. 209-214, 1992.

TERRAZZAN, E. A. Perspectivas para a inserção de física moderna na escola média. 1994. Tese (Doutorado) - USP, São Paulo. 